A. Pramesh Rao, G. Swarup and Gopal-Krishna, eds.

\title{
A WSRT Survey for HI Absorption at Moderate Redshifts
}

\author{
W.M. Lane and F.H. Briggs \\ Kapteyn Astronomical Institute, Postbus 800, 9700 AV Groningen, \\ The Netherlands
}

\begin{abstract}
We summarize results from an extensive search for $\mathrm{HI} 21 \mathrm{~cm}$ absorption at moderate redshifts. The survey was limited to systems with $\mathrm{Mg}$ II absorption at redshifts in the range $0.2<z<1.0$, which lie in front of bright radio quasars. Narrow band observations centered at the metalline redshifts were made with the UHF-high system and, when possible, the DZB correlator at the WSRT. Three new absorbers have been confirmed and several candidate absorbers identified. Optical depth limits of a few percent at velocity resolutions of about $6 \mathrm{~km} \mathrm{~s}^{-1}$ are obtained for most of the non-detections. The $21 \mathrm{~cm}$ search is complementary to similar damped Ly $\alpha$ surveys, and by combining information from both absorption lines we are able to better understand physical conditions in these high neutral column density absorbers. We find that the $21 \mathrm{~cm}$ objects represent a cold subset of high neutral column density absorbers, but are not necessarily the highest column density systems.
\end{abstract}

\section{Introduction}

Damped Ly $\alpha$ (DLA) lines are the spectral signatures of systems with high neutral column density (canonically, $N(\mathrm{HI}) \geq 2 \times 10^{20} \mathrm{~cm}^{-2}$ ). Such systems are the major contributors to the mass density of neutral gas at high redshifts $(z \approx 3)$. Although rare, several large optical surveys over the past fifteen years have greatly increased the number of such systems which are known at $z>2$ (cf. Wolfe, et al., 1986, Lanzetta, et al. 1991, Wolfe et al., 1995, Storrie-Lombardi et al. 1996). Attempts to identify these systems at lower redshifts, where the Ly $\alpha$ line falls in the UV regime have also been made, with varying success (cf. Lanzetta, et al., 1995, Rao and Turnshek, 1999). Consequently, nearly 100 DLA absorbers have now been identified over a wide range of redshifts. Despite the success of recent attempts to identify these systems, our understanding of the morphology and evolution of the host galaxies in which they arise remains poor.

$\mathrm{HI} 21 \mathrm{~cm}$ absorption features in radio spectra are expected to arise in gas at similar neutral column densities to those in the DLA line systems. Thus the radio regime offers a second method for identifying these neutral gas-rich systems. Due to limitations on most existing spectrometers, it is not possible to survey large amounts of redshift space in a time effective manner. It is, however, possible to preselect known low-ionization QSO absorption line systems, based on the presence of, for example, the MgII $\lambda \lambda 2796,2803$ doublet. A narrow band can be centered at the metal-line redshift to search for $21 \mathrm{~cm}$ absorption (Briggs 
\& Wolfe, 1983). This candidate selection can also be applied to UV surveys for DLA absorbers, where limited telescope time prohibits blind searches.

The $21 \mathrm{~cm}$ line provides information on the velocity dispersion of the gas, and, in combination with the DLA line, on the harmonic mean spin temperature, which is a measure of the relative amounts of warm and cold phase gas. Thus, in order to investigate the properties of DLA and $21 \mathrm{~cm}$ absorbing galaxies, comparable samples are needed of both types of absorbers. We present here the results from a large WSRT survey for redshifted intervening $21 \mathrm{~cm}$ absorbers, at redshift $0.2<z<1.0$. In addition, we present information on the lowest redshift DLA absorption system, which is found to be a $21 \mathrm{~cm}$ absorber as well.

\section{The Survey}

\subsection{Selection and Data}

The survey was limited to QSO absorption line systems which exhibit Mg II $\lambda \lambda 2796,2803$ absorption at redshifts in the range $0.2<z<1.0$. In order to facilitate the survey, the background QSOs were constrained to have a flux density of roughly $0.5 \mathrm{Jy}$ or greater at $1400 \mathrm{MHz}$ or $365 \mathrm{MHz}$. Known $21 \mathrm{~cm}$ absorbers were excluded, but systems were included regardless of whether they showed DLA absorption, did not, or had never been observed for Ly $\alpha$ absorption at all.

Narrow band observations centered at the metal-line redshifts were made with the UHF-high, 700-1200 MHz receivers at the Westerbork Synthesis Radio Telescope (WSRT). Although it requires a 12-hour integration to make a full synthesis with this 14 element E-W array, it was possible to achieve the desired spectral noise level in much shorter time periods for these bright QSOs, most of which are also unresolved. Therefore, integration times were determined purely by receiver response at the observing frequency and background source strength. Most of the survey was done with the DXB correlator, but when possible, the new DZB correlator was used. The benefit of the new correlator is the ability to make all of the 91 possible telescope combinations and the autocorrelations in each polarization, rather than a subset of 40 .

A total of $85 \mathrm{MgII}$ systems were observed during the first few months of 1997 and 1998. For 16 of these, rfi dominated the observation, leaving a final sample of $69 \mathrm{MgII}$ systems for which we have obtained $21 \mathrm{~cm}$ spectra. Standard calibration observations of either $3 \mathrm{C} 48,3 \mathrm{C} 147$, or $3 \mathrm{C} 286$ were used to calibrate flux levels and passbands. Reduction used standard routines in either AIPS, Newstar, or some combination of the two packages.

\subsection{Results}

We have identified 7 new candidate $21 \mathrm{~cm}$ absorbers for which we are currently in the process of obtaining followup observations. In addition, 3 new absorbers have been confirmed: a $z_{a b s}=0.2212$ system towards B0738+313. a $z_{a b s}=0.3127$ system towards B1127-145 (Lane et al. 1998), and one at a redshift $z_{a b s}=0.3941$ towards B0248+430. They are discussed individually below.

In a comparable UV survey, Rao and Turnshek (1999) find 8 DLA absorbers in a sample of $55 \mathrm{MgII}$ systems, over the same redshift range as the radio survey. 
This detection rate is considerably higher than our current detection level of 3 systems out of 69 , and even allowing for some of the $21 \mathrm{~cm}$ candidates to be real detections, will probably remain so. The low detection rate can be partially explained by the selection of the initial samples: Rao and Turnshek constrained their MgII sample to have equivalent widths greater than $0.3 \AA$, and in fact find that all but one of the detected DLA systems have widths greater than $0.6 \AA$. For the radio survey, no constraints on the MgII line widths were applied, and therefore many small equivalent width systems, unlikely to have associated high column density HI gas, are included.

\subsection{The Absorbers}

The $21 \mathrm{~cm}$ absorber at $z=0.3941$ towards B0248+430 is a multicomponent system, with at least 3 narrow deep features spanning about $35 \mathrm{~km} \mathrm{~s}^{-1}$ (Lane \& Briggs 2000). The individual features are unresolved in the finding spectrum. This particular system was included in the Rao and Turnshek (1999) survey, but they were unable to measure the Ly $\alpha$ absorption due to poor signal to noise. Using line parameters for the three components, the total column density in the system is $\mathrm{N}_{H I}=5.1 \pm 0.6 \times 10^{18} \mathrm{~T}_{s} \mathrm{~cm}^{-2} \mathrm{~K}^{-1}$, where $\mathrm{T}_{s}$ is the mean harmonic spin temperature of the gas.

The absorber at $z=0.3130$ towards B1127-145 is an extremely broad, multicomponent system, extending over a velocity range of more than $80 \mathrm{~km}$ $\mathrm{s}^{-1}$. In the DLA profile, the fitted neutral column density is $\mathrm{N}_{H I}=5.1 \pm 0.9 \times$ $10^{21} \mathrm{~cm}^{-2}$. There are 3 known galaxies near the QSO sightline which have measured spectroscopic redshifts similar to the redshift of the absorption (Lane et al. 1998). One of these three is visibly disturbed, and it is possible that the $21 \mathrm{~cm} /$ DLA absorption is arising at least partially in gas disturbed by galaxy interactions (cf. Rauch, Haehnelt, \& Steinmetz 1997; Haehnelt, Steinmetz, \& Rauch 1998).

The absorber at $z=0.2212$ towards $\mathrm{B} 0738+313$ (or OI 363) is a narrow, single component system (Lane, et al. 1998). With an optical depth at the line center of $9.9 \%$, the FWHM velocity is only $\Delta v=5.4 \pm 0.5 \mathrm{~km} \mathrm{~s}^{-1}$. From the DLA absorption feature, a column density for the system of $\mathrm{N}_{H I}=7.9 \pm 1.4 \times 10^{20}$ $\mathrm{cm}^{-2}$ is measured (Rao and Turnshek, 1998). Comparing the line parameters of the $21 \mathrm{~cm}$ absorption with the $\mathrm{N}_{H I}$ of the DLA line, a spin temperature of $\mathrm{T}_{s}=780 \pm 170 \mathrm{~K}$ is derived. This is similar to values found in other redshifted HI $21 \mathrm{~cm} /$ DLA absorption systems, despite being much higher than values measured within our Galaxy. Due to the narrowness of the $21 \mathrm{~cm}$ line it is possible to estimate the kinetic temperature of the absorbing gas directly, assuming that all of the line broadening is thermal rather than the result of bulk kinematical motions. The result is that $\mathrm{T}_{k}=630 \pm 120 \mathrm{~K}$, and thus within the errors, $\mathrm{T}_{s} \approx$ $\mathrm{T}_{k}$.

\section{The Lowest Redshift Damped Ly $\alpha$ Absorber}

The sightline towards B $0738+313$ contains a second DLA $/ 21 \mathrm{~cm}$ absorber, at the even lower redshift of $z=0.0912$ (Rao \& Turnshek 1998; Chengalur \& Kanekar 1999). The $21 \mathrm{~cm}$ absorption spectrum, taken with Arecibo, shows 2 very narrow and deep absorption components with FWHM velocities of $\Delta v_{1}=3.7$ and $\Delta v_{2}=$ 
$2.2 \mathrm{~km} \mathrm{~s}^{-1}$ (main and secondary component respectively) (Lane et al. 2000) Although the harmonic mean spin temperature calculated by comparison of the $21 \mathrm{~cm}$ lines to the damped $\mathrm{Ly} \alpha$ line is $\mathrm{T}_{s}=775 \pm 100 \mathrm{~K}$, the thermal kinetic temperatures of the two narrow components, calculated from their widths, are much lower: $T_{k} \leq 297 \pm 3$ and $\leq 103 \pm 10 \mathrm{~K}$ respectively. This is the first case of a redshifted absorption system for which $T_{k}$ is measured to be less than $\mathrm{T}_{s}$.

We interpret this result in the context of a two phase neutral gas model, in which the damped Ly $\alpha$ gas is sensitive to a significant neutral column density of warm phase gas as well as the cold phase gas of the narrow $21 \mathrm{~cm}$ lines. The $21 \mathrm{~cm}$ absorption spectrum is actually best fit by three line components; the two deep narrow components and a third broad and shallow component. The third component is interpreted as representing the warm phase gas with with $T_{k} \leq 5050 \pm 950 \mathrm{~K}$. The combined column density of the three $21 \mathrm{~cm}$ components is approximately equal to that derived from fits to the DLA line: $\mathrm{N}_{H I}=1.5 \pm 0.2 \times 10^{21} \mathrm{~cm}^{-2}$. The temperature of the two gas phases are consistent with temperatures measured in our own Galaxy (Kulkarni \& Heiles, 1988).

Acknowledgments. Support for W.M. Lane is provided by an Ubbo Emmius fellowship for graduate study at the Rijksuniversiteit Groningen.

\section{References}

Briggs, F.H., \& Wolfe, A.M. 1983 ApJ, 268, 76

Chengalur, J.N., \& Kanekar, N. 1999, MNRAS, 302, L29

Haehnelt, M.G., Steinmetz, M., \& Rauch, M. 1998, ApJ, 495, 647

Kulkarni, S.R., \& Heiles, C. 1988, in Galactic and Extragalactic Radio Astronomy, ed. G. Verschuur \& K. Kellerman (Heidelberg: Springer), 95

Lane, W.M., Smette, A., Briggs, F.H., Rao, S.M., Turnshek, D.A., \& Meylan, G. 1998, AJ, 116, 26

Lane, W. M., Briggs, F.H. 2000 in prep.

Lane, W.M., Briggs, F.H., \& Smette, A. 2000, ApJ, (astro-ph/9911142)

Lanzetta, K.M., Wolfe, A.M., Turnshek, D.A., Lu, L., McMahon, R.G., \& Hazard, C. 1991, ApJS, 77, 1

Lanzetta, K.M., Wolfe, A.M., Turnshek, D.A. 1995, ApJ, 440, 435

Rao, S.M., \& Turnshek, D.A. 1998, ApJ, 500, L115

Rao, S.M., \& Turnshek, D.A. 1999, preprint (astro-ph/9909164)

Rauch, M., Haehnelt, M.G., \& Steinmetz, M. 1997 ApJ, 481, 601

Storrie-Lombardi, L.J., McMahon, R.G., Irwin, M.J., \& Hazard, C. 1996, ApJ, 468,121

Wolfe, A.M., Turnshek, D.A., Smith, H.E., \& Cohen, R.D. 1986, ApJS, 61, 249

Wolfe, A.M., Lanzetta, K.M., Foltz, C.B., \& Chaffee, F.H. 1995, ApJ, 454, 698 